\title{
El crecimiento electoral de la derecha radical populista en Europa: parámetros ideológicos y motivaciones sociales
}

\author{
Joan Antón-Mellón y Aitor Hernández-CARR \\ Universidad de Barcelona \\ jantonmellon@ub.edu aitorcarr@gmail.com
}

Recibido: 25-02-2015

Aceptado: 08-02-2016

\begin{abstract}
La derecha radical populista se ha convertido en un actor político de primer orden en el continente europeo. El texto analiza el recorrido y las distintas fases que han llevado a este espacio político desde los márgenes del debate político a una centralidad conseguida tanto a través de su capacidad para ir ganando apoyo electoral como de incidencia en las agendas políticas de sus respectivos países. Asimismo, aborda el núcleo ideológico de estas formaciones, las temáticas que han centrado su capacidad de movilización política y el tipo de electorado que han conseguido aglutinar bajo sus siglas. Finalmente, se reflexiona también sobre el significado y el impacto del crecimiento de unas ideas y propuestas como las que representan la derecha radical populista.
\end{abstract}

Palabras clave: derecha radical populista; nueva extrema derecha; extrema derecha; populismo; Europa.

\section{The Electoral Rise of Populist Radical Right Parties in Europe: Ideological Parameters and Social Motivations}

\begin{abstract}
Populist radical right parties have become major political actors in Europe. This paper analyses the path and the different phases that have led them from the fringes of public debate to their present significance, which is based on their capacity to attract electoral support and influence the political agendas in their respective countries. Besides, an analysis of the core ideological beliefs of these parties, and of the topics on which their mobilization capacity rests, is provided, as well as of the type of voters that are attracted by them. Finally, the authors discuss the meaning and impact of the growing popularity of the ideas and proposals put forward by the populist radical right parties.
\end{abstract}

Key words: populist radical right parties; new extreme right; extreme right parties; populism; Europe.

\section{Referencia normalizada}

Antón-Mellón, J. y A. Hernández-Carr (2016): "El crecimiento electoral de la derecha radical populista en Europa: parámetros ideológicos y motivaciones sociales", Política y Sociedad, 53 (1), pp. 17-28.

Sumario: Introducción. 1. De los márgenes al centro del debate político. 2. El núcleo ideológico de la derecha radical populista. 3. Los ejes centrales de su movilización política. 4. ¿Quién vota a los partidos de derecha radical populista?. 5. Conclusiones. 6. Bibliografía. 


\section{Introducción}

A partir de los años ochenta del siglo XX los partidos comúnmente conocidos como "de extrema derecha" han desempeñado un creciente y relevante papel en la vida política europea. Tanto por sí mismos como por la influencia que ejercen en las agendas y en los programas de otros partidos de los sistemas de partidos en los que actúan (Van Spanje, 2010). Los resultados obtenidos por algunas de estas formaciones en las elecciones europeas de mayo del 2014 han situado este fenómeno en el centro de la agenda mediática europea ${ }^{1}$. Si bien en el pasado partidos situados en este espacio político han recibido una gran atención mediática, la percepción que estos partidos avanzaban en gran parte del continente europeo, junto a las sonadas victorias en países como Francia, Dinamarca o Reino Unido, han hecho saltar todas las alarmas. Sus resultados han generado una enorme atención mediática y han abierto todo tipo de interrogantes sobre los antecedentes, motivos y consecuencias de este éxito.

El siguiente texto expone las principales características de una familia de partidos que consideramos debe ser diferenciada de la extrema derecha tradicional y que ha sido descrita como una "nueva extrema derecha" o "derecha radical populista", entre otros términos (Mudde, 2007). Para ello analizaremos en primer lugar la trayectoria electoral y política de este sector político desde los años ochenta hasta la actualidad. Seguidamente abordaremos los principios ideológicos nucleares de estos partidos, las temáticas a partir de las cuales han desarrollado su movilización política, y, expondremos brevemente el electorado que estos partidos han conseguido aglutinar. Finalmente, concluiremos con una reflexión sobre el impacto de estos partidos en los sistemas políticos y en el conjunto de las sociedades europeas actuales.

\section{De los márgenes al centro del debate político}

La trayectoria de esta familia de partidos, desde su irrupción en los años 80 hasta la actualidad, puede ser comprendida alrededor de tres grandes etapas. Durante los primeros años, las formaciones de nueva extrema derecha ocuparon posiciones claramente secundarias dentro del escenario político de sus respectivos países. Pese a atraer una gran atención mediática, los porcentajes de voto que obtenían no acostumbraban a pasar del $10-15 \%$, y existía un claro posicionamiento en el resto de partidos, en el sentido que no eran socios aceptables para establecer pactos o coaliciones de gobierno. Estaban, por tanto, en los márgenes del sistema político.

Esta situación empezó a cambiar a lo largo de los años noventa. La irrupción de un número aún mayor de partidos de nueva extrema derecha, el progresivo crecimiento electoral de estos y la estabilidad dentro de sus respectivos sistemas políticos, fueron minando la consideración de partidos marginales y/o no aptos para entrar en pactos y coaliciones de gobierno. La entrada del Partido de la Libertad austríaco, la Liga Norte italiana y el Partido Popular Danés en los respectivos gobiernos, junto con el inédito

${ }^{1}$ Un breve repaso de los resultados de la extrema derecha en las elecciones europees del 2014 puede encontrarse en http:/www.lamarea.com/2014/05/26/resultados-de-la-extrema-derecha-en-europa-por-paises/ 
acceso del Frente Nacional a la segunda vuelta de las presidenciales francesas de 2002, marcaron un punto de inflexión. Pese a que la derecha radical populista se estaba acercando al centro del escenario político, se produjo una considerable reacción contra estos hechos. En el caso del Frente Nacional de Jean-Marie Le Pen, su éxito electoral fue respondido con movilizaciones masivas en la calle y el reforzamiento del cordón sanitario alrededor suyo. En el caso del FPÖ, desde la Unión Europea se llegaron a imponer sanciones al gobierno austriaco como medida de represalia por la presencia de este partido. Unas medidas que finalmente hicieron caer la coalición entre el FPÖ y los conservadores de Wolfgang Schüssel.

La tercera etapa sería el contexto de crisis económica e institucional en que se encuentra inmersa la Unión Europea desde el periodo 2007-2008 hasta la relevante victoria de algunos de estos partidos en las elecciones Europeas de 2014. Es importante apuntar que, tal y como hemos mostrado, el crecimiento y el arraigo de estos partidos es previo a la crisis económica. Asimismo, no se puede afirmar que en los últimos años la extrema derecha haya crecido en todos los países europeos ${ }^{2}$. Sin embargo, el contexto de crisis sí ha ido abriendo una ventana de oportunidad para eventos difícilmente imaginables diez años atrás. Nos estamos refiriendo a la sonada irrupción de un partido abiertamente neonazi como Amanecer Dorado en Grecia (9,3\% de los votos) o la contundente victoria del Frente Nacional a las elecciones europeas de mayo de 2014 $(25,4 \% \text { de los votos })^{3}$.

Dentro de la fase actual de crecimiento de la derecha radical populista, consideramos que hay tres elementos especialmente destacados. El primer elemento ya comentado son las victorias electorales obtenidas por vez primera por estos partidos en países como Francia, Dinamarca o Inglaterra. Pese a que dicha victoria se ha producido en unas elecciones europeas, una contienda tradicionalmente más propicia para estos partidos, el hecho de ganar las elecciones es una muestra de fuerza de cara al conjunto del electorado y el resto de formaciones políticas y abre nuevas perspectivas en cuanto a las aspiraciones electorales de estos partidos en elecciones legislativas o presidenciales. Mención especial merecen los resultados del Frente Nacional. Esto es así por la solidez de sus resultados y por el hecho de que el Frente Nacional ha tenido históricamente un papel preponderante dentro de lo que es la nueva extrema derecha europea. No en vano, fue su irrupción en las elecciones europeas de 1984 lo que provocó un "efecto contagio" llevando a otros partidos, como el Partido de la Libertad austriaco (FPÖ), a imitar su discurso político (Rydgren, 2005). Desde entonces, a pesar de no ser siempre

${ }^{2}$ Sobre la necesidad de extremar las precauciones a la hora de afirmar que la extrema derecha está creciendo en todo el continente europeo a raíz de la crisis económica véase este texto de Cas Mudde: http://blogs.lse.ac.uk/europpblog/2013/08/22/contrary-to-popular-opinion-europehas-not-seen-a-rise-in-far-right-support-since-the-start-of-the-crisis/

${ }^{3}$ La solidez de sus resultados puede comprobarse si se tiene en cuenta que en las elecciones europeas el FN fue el partido más votado en prácticamente todos los departamentos territoriales, el partido más votado entre los jóvenes (30\%), los obreros (43\%) y los parados (37\%). Sobre estas cifras véase http://internacional.elpais.com/internacional/2014/05/25/actualidad/1401042233_633658.html 
el partido que ha obtenido unos porcentajes de voto más elevados, el FN ha tenido un papel clave en el escenario político de la extrema derecha europea. En este sentido, sus buenos resultados tienen un efecto que va más allá de territorio francés.

En segundo lugar, la crisis económica parece haber abierto la puerta del éxito electoral a formaciones de extrema derecha tradicional o abiertamente neo-fascistas, que difícilmente hubiesen podido obtener dichos resultados en un contexto precrisis. Siendo la irrupción de Aurora Dorada el ejemplo más claro de ello. Estamos, por tanto, ante la posibilidad que partidos cuyo imaginario y formas de movilización política parecían totalmente superadas, cobren protagonismo en el terreno electoral y revigoricen el espacio político de la extrema derecha tradicional. El tercer elemento a destacar es la creciente voluntad entre algunos de estos partidos por coordinarse y ser más efectivos a la hora de influir en la agenda política europea. En este sentido, destaca especialmente el pacto para tratar de formar un grupo parlamentario propio entre partidos de tanto peso como el Frente Nacional francés, el Partido de la Libertad austriaco, el Vlaams Belang flamenco, el Partido de la Libertad austriaco y la Liga Norte italiana. En este sentido, el pacto rompe algunas de las divisiones entre estos partidos que habían dificultado su coordinación y colaboración. Especialmente importante es la presencia del Partido de la Libertad de Geert Wilders, dado que hasta el momento se había mantenido alejado de partidos como el Frente Nacional francés por considerarlos excesivamente "radicales"4.

\section{El núcleo ideológico de la derecha radical populista}

Las similitudes, pero también las diferencias, entre estos partidos han llevado a un extenso debate sobre si estamos o no ante una nueva familia de partidos y cuáles son las variables centrales de dicha familia de partidos (De Lange y Mudde, 2005). Un primer hito de dichos debates consistió en la distinción efectuada por el politólogo italiano Piero Ignazi entre "extrema derecha tradicional" y "extrema derecha postindustrial" o "nueva extrema derecha" (Ignazi, 2003). Para este autor el factor decisivo de distinción y que permite hablar de dos familias de partidos consistiría en la no ruptura de los primeros con los vínculos fascistas, factor que les ha conducido a su creciente marginalización política o su pervivencia en reducidos círculos de creyentes que se retroalimentan principalmente en realidades virtuales ciberfascistas. Siguiendo la influyente propuesta de Cas Mudde, puede decirse que los partidos de derecha radical populista constituyen una nueva familia de partidos, diferente de la extrema derecha porque la derecha radical populista es “(...) (nominalmente) democrática, aunque se opongan a algunos valores fundamentales de las democracias liberales, mientras que la extrema derecha es en esencia anti-democrática, al oponerse al principio fundamental de la soberanía del pueblo" (Mudde, 2007:31). Por ello, a pesar, de los evidentes vínculos histórico-ideológicos entre la extrema derecha tradicional y estas

${ }^{4}$ Sobre esta alianza véase http://internacional.elpais.com/internacional/2013/11/13/actualidad/1384343925_371743.html 
"nuevas" formaciones políticas, deben ser consideradas como dos familias de partidos diferentes (Goodwin, 2007).

El "núcleo ideológico" compartido por estas formaciones políticas serían el nacionalismo, populismo y autoritarismo, como variables ideológicas centrales y compartidas o ideas-fuerza/concepciones nucleares específicas (Casals, 2003; Mudde, 2007; Antón-Mellón, 2007). Respecto al nacionalismo diferentes autores (Betz, 2004; Rydgren, 2007; Mudde, 2007) consideran que éste es el factor decisivo aunque valdría la pena matizar que son planteamientos que no solo deben ser calificados de nacionalistas sino de ultranacionalistas, de ahí que estos mismos autores hayan adoptado el término Nativismo (Betz, 2007; Mudde, 2007). El Nativismo puede definirse como: “(...) una ideología que sostiene que los estados deberían ser habitados exclusivamente por miembros del grupo nativo ("la nación" y que los elementos no-nativos (personas e ideas) son fundamentalmente una amenaza para un estado-nación homogéneo." (Mudde, 2007:19).

Nacionalismo/ultranacionalismo nativista que debe ser relacionado, necesariamente, al populismo como estilo de actuación política, en una forma novedosa y específica de populismo tal y como apunta el profesor Taguieff (2007): "nacional-populismo", al imbricarse la clásica invocación populista de "los de arriba" confrontados contra "los de abajo" con la llamada palingenésica (regenerativa) a la comunidad nacional amenazada por la inmigración, una clase política corrupta y traidora y la mundialización. En cita del propio autor: "El nacional-populismo de Le Pen ofrece simultáneamente una voz de protesta y de identidad. Apela al ethnos (la nación étnicamente pura) y al demos (las clases populares "incorruptas"). Apela al hombre pequeño frente a los grandes hombres y al pueblo en relación a una identidad supuestamente amenazada. " (Taguieff, 2004; en Perrineau, 2005:23).

El populismo es un método o estilo de actuación política que se utiliza para lograr un particular tipo de movilización social y política, normalmente en situaciones de crisis económica $\mathrm{y}$, sobre todo, de crisis política por procesos de deslegitimación de las élites políticas. En el caso que nos ocupa se utiliza, reiterativamente, una estructura de argumentación narrativa en torno a cuatro ejes: paro, inmigración, inseguridad y corrupción. Estilo populista como palanca para acceder al poder y hacer creíble un discurso nacional-populista todo lo autoritario que un sistema formalmente democrático pueda permitir, multiplicado mediáticamente dicho discurso por fuertes liderazgos carismáticos (Eatwell, 2007). El disponer o no de un líder carismático que domine los medios de comunicación de masas, puede ser un factor decisivo en el éxito de estos partidos (el fracaso de B. Mégret y su escisión del FN frente a Jean-Marie Le Pen es ilustrativo).

La clave ideológica del populismo radica en el uso político del término pueblo como comunidad nacional, de ahí que sea tan relevante definir legalmente quién pertenece a la nación. Un "pueblo" idealizado, constituido/imaginado por un conjunto de ciudadanos llanos poseedores de un sentido común político innato, justo y sabio que no pueden emplear por la corrupción de las élites. Ante esta situación el "pueblo" debe tomar el poder y con ello todos los problemas sociales desaparecerán. Un extenso movimiento nacional armonicista, suprapartidista y supraclasista lo logrará. Aunque, como es obvio, 
estas creencias sobre la bondad intrínseca del ciudadano llano y la maldad consustancial de las élites es un demagógico reduccionismo usado por los políticos profesionales populistas para lograr una extensa base social popular. Igual que en los años treinta, la demagogia compensa las contradicciones y la ambigüedad doctrinal es un factor que atraviesa las barreras ideológicas y sociológicas, planteando soluciones simples a temas complejos. La apelación al "pueblo" se da en un contexto de transformaciones sistémicas conflictivas (crisis económica) deslegitimación y desafección política y hegemonía ideológica de la soberanía popular, de ahí que los partidos populistas de derecha radical levanten la bandera de la "auténtica democracia". Por ello, adelantando aquí futuras conclusiones, pensamos que el déficit democrático sistémico en el que actúan dichos partidos es el factor explicativo causal más importante que nos permite entender el surgimiento, expansión y, sobre todo, consolidación, de dichos partidos.

Finalmente en este apartado analizaremos el tercer factor característico (tras haber reflexionado sobre los factores nacionalista y populista) de los partidos estudiados, el autoritarismo. En este sentido el brillante concepto de Roger Griffin (2000): Liberalismo Etnocrático, explicita muy bien las propuestas de dichos partidos. Las viejas culturas europeas autoritarias de derecha radical de los años treinta, tras su derrota en 1945, se han visto obligadas a reconvertirse para ser admitidas por el sistema, se han visto obligadas a asumir el liberalismo con todas sus consecuencias: alternancia del poder, renuncia a la violencia, imperio de la ley etc. etc. Aceptan la democracia parlamentaria y sus reglas pero hacen una lectura autoritaria- conservadora de ésta. Una lectura que fuerza los límites de los valores democráticos y que pretende la exclusión permanente y legalmente establecida de una parte de la población a la cual se le niega la nacionalidad y con ella unos plenos derechos. Un sistema de discriminación institucionalizado y validado democráticamente (Antón-Mellón, 2007). En este sentido diferentes autores (Griffin , 2000; Betz, 2002; Rodríguez, 2006) han señalado que estos partidos tienen una doble influencia negativa en la cultura democrática europea, por si mismas en cuanto a su capacidad de gobernar y desarrollar sus planteamientos y por su capacidad de influir en las agendas de otras formaciones políticas que adopten parte de sus enfoques, en este sentido la adopción de determinadas políticas étnicamente discriminatorias por parte de los partidos políticos franceses (desde la derecha a la izquierda socialdemócrata) son muy ilustrativas. No se trata de que las libertades democráticas estén en peligro de ser anuladas, como en los años treinta, sino que Europa admita que pueda ser democrático la existencia de ciudadanos de primera y ciudadanos de segunda: una xenofobia legalizada e institucionalizada para que los beneficios sociales del sistema sólo alcancen a los que legalmente definamos como nacionales, excluyendo a los "otros" y garantizando así el consenso políticos necesario para poder garantizar la estabilidad de unas sociedades clasistas que priman, sistémicamente, las rentas del capital sobre las del trabajo, tanto en coyunturas expansivas como en las recesivas. Griffin expone que en las circunstancias históricas actuales los partidos de derecha radical populista son más inquietantes y peligrosos que las nostálgicas organizaciones de extrema derecha puesto que: "(...) el liberalismo etnicista ha reemplazado al fascismo como la forma de derecha radical populista mejor adaptada a las realidades del mundo moderno" (Griffin, 2000:19). 


\section{Los ejes centrales de su movilización política}

Las temáticas usadas por estos partidos como motor de su movilización política han ido variando con el pase del tiempo y según las especificidades nacionales. No obstante, dos temas han sido centrales en la construcción del imaginario y los discursos políticos de los partidos de derecha radical populista un discurso populista anti-establishment y un discurso de rechazo frontal a la población extranjera. Tal y como señala Rydgren (2005) y analizaremos a continuación, la exitosa movilización política de estas formaciones se ha edificado a partir de "un etno-nacionalismo basado en el "racismo cultural" (la doctrina conocida como "etno-pluralista") y una retórica populista (pero no antidemocrática) anti-establishment político"

\subsection{Xenofobia anti-inmigrante}

El odio al extranjero: los inmigrantes se ha convertido en las señas de identidad de los partidos estudiados, constituyendo, en la actualidad, el eje central de sus discursos. Eje central ideológico, factor de movilización social y banderín de enganche políticoelectoral. Discursos ideológicos xenófobos que se producen en un contexto histórico de gradual pérdida de soberanía de los estados-nación, globalización: económica y cultural, crisis económica y fluctuantes flujos de población inmigrante. Produciendo, todo ello, malestar y crisis de identidad que buscan como demandas respuestas políticas u ofertas que den alternativas a los miedos, inseguridades y angustias sociales existentes. El inmigrante, el "otro", el que es diferente a mí se convierte en el cabeza de turco, el "gran culpable" en el que hacer recaer todas las culpas. Máxime si tenemos presente que la cultura política referencial de estas organizaciones proviene de la extrema derecha/derecha radical ultranacionalista según la cual los protagonistas de la historia son las comunidades nacionales étnicamente homogéneas, de ahí su frontal rechazo a los inmigrantes y al multiculturalismo.

Habiéndose producido un cambio cultural y discursivo importante: el viejo racismo biológico de los años treinta ha sido substituido por el nuevo racismo cultural (Wieviorka, 1992) o "racismo diferencialista" (Taguieff, 1993-1994). La "raza" es substituida por la "cultura" como factor de clasificación y de división entre individuos y grupos, viendo en la cultura un factor identitario decisivo y prácticamente estático. De esta forma las naciones son fruto de la historia, las tradiciones y, sobre todo, unos determinados rasgos culturales esencialistas que se transmiten de generación en generación y, por ello, los inmigrantes, ajenos a todos estos factores, son inasimilables, son un cuerpo extraño en la comunidad nacional que entorpece su camino. A partir de estas creencias los partidos de derecha radical populista afirman que una "excesiva" llegada de población inmigrante desnaturaliza la identidad cultural de la comunidad y, consiguientemente, pone en peligro a la propia nación. Así impedir la plena integración de los extranjeros en el cuerpo nacional es un deber ineludible de los "patriotas". Como es obvio cuanto más diferenciadas sean los factores culturales entre los grupos inmigrantes y las comunidades autóctonas mayor será la discrepancia y el rechazo, siendo las poblaciones de cultura islámica el paradigma máximo, factor que se retroalimenta ante las minoritarias actitudes de sectores radicalizados en las propias poblaciones musulmanas. 
El racismo diferencialista se ha desarrollado en un contexto (post 1945) de hegemonía de los valores democráticos, por lo que su argumentario es todo lo sofisticado y alambicado necesario para ser operativo. Se afirma que individuos y culturas no son desiguales sino diferentes y que la diversidad cultural es enriquecedora (lo que es cierto), por lo que es imprescindible preservar esa diversidad. Defender la identidad nacional propia, concluyen categóricamente, es el medio idóneo de conservar esa diversidad cultural, legitimando, de esta forma, las propuestas excluyentes, las actitudes xenofóbicas y el liberalismo etnocrático: "Austria primero" afirmaban los pasquines electorales del FPÖ austríaco; "hundamos a cañonazos los barcos de los inmigrantes ilegales" gritaba en los mítines Humberto Bossi de la Lega Nord italiana,

Como expone la profesora Stolcke (1995) el racismo diferencialista presupone que diferentes culturas no pueden coexistir en un mismo espacio público estatal (en contra de múltiples experiencias históricas), ya que se parte de la creencia de que la coexistencia conducirá, necesariamente, al conflicto. Lo cual significa creer que la xenofobia es un comportamiento natural en los seres humanos y que los individuos son solo solidarios con los de su grupo y hostiles a otros grupos. En última instancia dicha creencia se fundamenta en una visión de los seres humanos como seres intrinsicamente desiguales, agresivos y territorializados. Con el factor añadido que a la lucha por la preservación de las diferentes culturas nacionales debe añadirse la defensa de la cultura occidental/ europea en su conjunto, amenazada por culturas extrañas y desnaturalizadoras de sus auténticas esencias.

\subsection{Argumentario anti-establishment}

Los partidos de derecha radical populista se presentan ante la opinión pública no como partidos clásicos sino como movimientos nacionales suprapartidistas, creen estar más allá de la lucha ideológico-partidista al uso ya que, en su opinión, reflejan al conjunto del "pueblo" a la "nación auténtica". Nación/pueblo traicionada por politicastros que solo defienden sus propios espúreos intereses, por esa razón las organizaciones políticas estudiadas plantean un "nosotros" y "ellos" especial, "Ellos" son todos los demás, sin matizaciones de ningún tipo: o son élites corruptas o defienden sus intereses. "Los de abajo" contra "los de arriba" y, naturalmente, los partidos de derecha radical populista son los partidos defensores de "los de abajo".

Como expone Laclau (2005) el populismo debe ser considerado como una serie de recursos discursivos que pueden ser utilizados de diversas maneras, un estilo de comunicación política (Jagers y Walgrave, 2007), una estructura de argumentación narrativa (como previamente apuntábamos). En este caso al servicio instrumental del reciclaje oportunista de la derecha radical europea, que ha tenido en las inmigraciones masivas del siglo XX y XXI a Europa la tabla de salvación de su marginación política tras la derrota del fascismo en 1945. Las miserias de la postmodernidad han establecido una ventana de oportunidad política que estos partidos han sabido aprovechar con mayor o menor fortuna como, a su vez, en los años treinta del pasado siglo los partidos fascistas la tuvieron ante las miserias de la modernidad. 
El "pueblo", la "nación auténtica" está doblemente amenazada por la traición de las élites y por la gradual desnaturalización ante la masiva llegada de inmigrantes. A los primeros les trae sin cuidado lo segundo por lo que derrotar al establishment es sentar las bases para solucionar todos los problemas mediante criterios de "preferencia nacional". Todos los resentimientos sociales se canalizan en lo que se ha denominado un doble movimiento retórico (Betz, 2004:44): contra las élites y contra los inmigrantes.

El factor clave para lograr una exitosa movilización populista es lograr legitimidad democrática consiguiendo que la sociedad olvide y/o no tenga en cuenta los vínculos con los idearios de derecha radical del primer tercio del siglo XX (Rydgren, 2007), de ahí que se ensalce, reiteradamente, la soberanía popular, la auténtica democracia y el "pueblo" como sujeto político decisivo y decisor para el futuro de la nación. Reveladoramente el rechazo a las élites políticas es total, en numerosas ocasiones también a las élites culturales y en mucha menor medida a las élites económicas. Como máxime se denuncia un capitalismo opresor que siempre es el internacional, salvándose demagógicamente el nacional y sin efectuar el mínimo análisis sobre las imbricaciones de ambos.

\section{4. ¿Quién vota a los partidos de derecha radical populista?}

A partir del análisis de los resultados de un conjunto de investigaciones empíricas puede afirmarse, de forma fundamentada, que ha habido una evolución común del perfil socioeconómico del electorado de los referidos partidos. El punto de partida electoral de los años ochenta y primeros noventa era un voto mayoritariamente de clase media, a partir de esas fechas se produce una gradual "obrerización" del electorado (Evans y Ivaldi, 2002; Lubers et al., 2002; Ignazi, 2003), la suma de ambos sectores (con predominio del segundo) explicaría su creciente despegue (Ivarsflaten, 2005).

Por lo que se refiere a los votantes de clase media fundamentalmente se trata de comerciantes, pequeños empresarios y trabajadores autónomos (Evans, 2005), población laboral englobada en las categorías de auto-empleados y que han sido denominados por Ignazi (2003) de clase media "tradicional". Son, en su mayor parte, exvotantes de formaciones políticas conservadoras que se han radicalizado hacia la derecha por temas concernientes a la inmigración, la inseguridad y los temas identitarios. El descontento por la respuestas políticas a estos problemas de los partidos tradicionales de derecha habría llevado a estos votantes a respaldar opciones más radicales (Ignazi, 2003).

El voto obrero de estas formaciones políticas proviene de trabajadores por cuenta ajena no cualificados, trabajadores de cuello blanco de bajo nivel y parados (Lubbers et al., 2002; Mudde, 2007). Las investigaciones de la profesora Mayer nos muestran que dichos electores mayoritariamente son jóvenes (varones) (Givens, 2004) que no se consideran ni de izquierdas ni de derechas (ninistas), de escasa politización y huérfanos de adscripción ideológica, si a ello añadimos su poca formación (Mayer, 2003; Evans, 2005) y el desempleo (Mény y Surel, 2000) estas serían las claves explicativas.

Las posibles contradicciones entre los hipotéticos intereses de esos dos grupos de votantes serían resueltas mediante la canalización de los resentimientos sociales contra inmigrantes y élites. Se trataría de un "pensamiento mágico" que evitaría entrar a fondo 
en la elaboración de propuestas y programas de complicados análisis económicos tanto de ámbito nacional como internacional. Por ello normalmente los electores de estos partidos son individuos jóvenes (con menor lealtad electoral a partidos tradicionales) escasamente politizados e ideologizados (salvo los recalcitrantes y creyentes votantes/ militantes de derecha radical), individuos éstos que se motivan políticamente por actitudes mucho más primarias: "excesivo" número de inmigrantes y consiguientes cambios en el paisaje urbano, degradación del nivel de vida por la crisis, corrupción, aumento de las desigualdades, inseguridad, protesta anti-establishment. Factores todos ellos en los que pueden coincidir diferentes grupos sociales, incluso "perdedores" y "ganadores" de los procesos de la globalización.

\section{Conclusiones}

Como se ha intentando describir y explicar el auge electoral de los partidos populistas de derecha radical en Europa certifica nuevas e inquietantes realidades sociológicas y socioeconómicas. A relevantes cambios en el modelo productivo, consecuencia de la globalización y del momento histórico existente en la revolución científico-técnica, les corresponden cambios ideológicos y políticos. Surgen nuevas ofertas políticas para satisfacer nuevas demandas. En situaciones de crisis renacen las ideologías, se necesitan respuestas políticas a situaciones social, económica o políticamente agobiantes: crisis económica, desempleo, aculturación/mundialización cultural, inmigraciones masivas, pérdida gradual de soberanía de los Estados Nación, globalización de la inseguridad por terrorismos y crimen organizado, problemas identitarios, recortes en la desmercantilización lograda por los Estados de Bienestar, desafección política, etc. etc. Factores de entre los cuales cabe destacar la reorganización del sistema productivo a escala mundial (con la pérdida de su valor de mercado de los sectores obreros europeos no cualificados), la hegemonía de la agenda política neoliberal, la crisis de modelo de la izquierda clásica (socialdemocráta o comunista) y la consiguiente pérdida de capacidad de seducción social y socialización de la cultura obrera de izquierdas.

Todo ello en un contexto europeo de hegemonía de los valores democráticos, de ahí que los partidos estudiados levanten la bandera de la "auténtica democracia", secuestrada, según ellos, por élites corruptas y cosmopolitas ajenas a la defensa de la nación o de la federación de naciones: Europa. Sus propuestas "liberal-etnocráticas son una auténtica prueba de resistencia de los valores e instituciones democráticas que han sustentado a los paises europeos tras su victoria sobre el fascismo. Europa o profundizará en sus valores e instituciones democráticas o escuchará los cantos de sirena de la xenofobia/preferencia nacional

El Frente Nacional en Francia, el Vlaams Belang en Bélgica o el partido de la Libertad austríaco, por ejemplo, coinciden en levantar la bandera de la xenofobia, de las identidades nacionales/europeas y de la inseguridad ciudadana, capitalizando profundos descontentos sociales y sumando, así, minoritarios votos ideológicos de adhesión con otros, más mayoritarios, de protesta social o de búsqueda de una nueva identidad política. El fenómeno es europeo, estos partidos son manifestaciones locales 
de realidades globales: democracia avanzada o neo-barbarie, Tertia non datur (no hay una tercera vía).

\section{Bibliografía}

Antón-Mellón, J. (2007): "La cultura e ideología política del neopopulismo en Europa occidental: MNR/FN (Francia), FPÖ (Austria) y Lega Nord (Italia)", en M.A. Simón (ed.), La extrema derecha en Europa desde 1945 a nuestros dias, Madrid, Tecnos.

Betz, H-G. (2002): Exclusionary populism in Austria, Italy and Switzerland. Disponible en:

http://www.extremismus.com/forschung/english/english.html.

Betz, H-G. (2004): La droite populiste en Europe. Extrême et démocrate?, Paris, Autrement.

Betz, H-G. (2007): "Contra el "totalitarismo verde": nativismo antiislámico en los populismos radicales de derecha en Europa occidental", en M.A. Simón, ed., La extrema derecha en Europa desde 1945 a nuestros días, Madrid, Tecnos, pp. 105-130.

Casals, X. (2003): Ultrapatriotas. Extrema derecha y nacionalismo de la guerra fría a la era de la globalización, Barcelona, Crítica.

De Lange, S. y C. Mudde (2005): "Political extremism in Europe", European Political Science, 4 (4), pp. 476-88.

Eatwell, R. (2007): "Hacia un nuevo modelo de liderazgo carismático de derecha", en M.A. Simón, ed., La extrema derecha en Europa desde 1945 a nuestros días, Madrid, Tecnos, pp. 19-38.

Evans, J. (2005): "The dynamics of social change in radical right-wing populist party support", Comparative European Politics, 3, pp. 76-101.

Evans, J. y G. Ivaldi (2002): "Les dynamiques électorales de l'extrême droite européenne", Revue Politique et Parlementaire, 1019, pp. 67-68.

Givens, T. (2004): “The radical right gender gap", Comparative Political Studies, 37 (1), pp. 30-54.

Goodwin, M. (2007): "Grandpa's fascism and the new kids on the block: contemporary approaches to the dark side of Europe", Ethnopolitics, 6 (1), pp. 145-54.

Griffin, R. (2000): "Interregnum or endgame? Radical right thought in the 'Post-fascist' era", The Journal of Political Ideologies, 5 (2), pp. 163-78.

Ignazi, P. (2003): Extreme right parties in Western Europe, Oxford, Oxford University Press.

Ivarsflaten, E. (2005): “The vulnerable populist right parties: no economic realignment fuelling their electoral success", European Journal of Political Research, 44, pp. 465-92.

Jagers, J. y S. Walgrave (2007): "Populism as political communication style: an empirical study of political parties' discourse in Belgium", European Journal of Political Research, 46 (3), pp. 319-45.

Laclau, E. (2005): La razón populista, Buenos Aires, Fondo de cultura económica.

Lubbers, M., M. Gijsberts y P. Scheepers (2002): "Extreme right-wing voting in Western Europe", European Journal of Political Research, 41, pp. 345-78. 
Mayer, N. (2003): "Que reste-t-il du vote de classe? Le cas français", Lien social et Politiques, 49, pp. 101-11.

Mény, Yves e Y. Surel (2000): Par le peuple, pour le peuple. Le populisme et la démocratie, Paris, Fayard.

Mudde, C. (2007): Populist radical right parties in Europe, Cambridge, Cambridge University Press.

Perrineau, P. (2005): “Does Lepenism exist without Le Pen?", en X. Casals (ed.), Political survival on the extreme right. European movements between the inherited past and the need to adapt to the future, Barcelona, Institut de Ciències Polítiques i Socials.

Rodríguez, J.L. (2006): "De la vieja a la nueva extrema derecha (pasando por la fascinación por le fascismo)", Historia Actual Online, 9. Disponible en: http://www.historia-actual.com/hao/pbhaoabs. asp $2 \mathrm{idi}=\mathrm{ESP} \& \mathrm{pgt}=2 \& \mathrm{pid}=4 \& \mathrm{pbl}=\mathrm{HAO} \& \mathrm{vol}=1 \& \mathrm{iss}=9 \& \mathrm{cont}=9[$ Consulta: $20 \mathrm{de}$ enero de 2008].

Rydgren, J. (2005): "Is extreme right-wing populism contagious? Explaining the emergence of a new party family", European Journal of Political Research, 44 (3), pp. 413-37.

Rydgren, J. (2007): "The sociology of the radical right", Annual Review of Sociology 33, pp. 241-62.

Stolcke, V. (1995): "Talking culture: new boundaries, new rhetorics of exclusion in Europe", Current Anthropology, 36 (1), pp. 1-24.

Taguieff, P-A. (1993/1994): "From race to culture: the New Right's view of european identity", Telos, 98-99, pp. 99-125.

Taguieff, P-A. (2007): “Interpretar la ola populista en la Europa contemporánea: entre resurgencia y emergencia", en M.A. Simón (ed.), La extrema derecha en Europa desde 1945 a nuestros días, Madrid, Tecnos, pp. 39-66.

Van Spanje, J. (2010): "Contagious parties: anti-immigration parties and their impact on other parties' immigration stances in contemporary western Europe", Party Politics, 16, pp. 563-586.

Wieviorka, M. (1992): El espacio del racismo, Barcelona, Paidós Ibérica. 


\title{
Transformaciones contemporáneas de la relación entre política, cultura y religión en Europa occidental. Un apunte para el caso español
}

\author{
Alfonso Pérez-Agote \\ Universidad Complutense de Madrid \\ pereza@cps.ucm.es
}

Recibido: 25-02-2015

Aceptado: 08-02-2016

\section{Resumen}

Según Tilly dos leyes definen el proceso de transformación de las sociedades europeas occidentales desde la Paz de Westfalia hasta finales del siglo XX: la de la progresiva homogeneidad interna de cada una y la de la progresiva heterogeneidad entre todas ellas. La homogeneización cultural interna primero ocurre en relación a los grupos étnicos coexistentes dentro del territorio del Estado. La fase siguiente de homogeneización tendría lugar en relación con las poblaciones inmigradas tras la Segunda Guerra Mundial; proceso que siguió diversos modelos según los países, pero que la crisis de los años setenta, la del petróleo, mostró su general falta de éxito. Durante este último cuarto del siglo XX, estas sociedades se ven así atravesadas por dos lógicas globales, progresivas y contradictorias: la de homogeneización cultural a escala planetaria (ya no de cada sociedad) y la de re-creación cultural llevada a cabo por la población de origen inmigrante, que, responden al fracaso integrador con la búsqueda de fuentes de estima personal y social en su universo cultural-religioso. Este trabajo trata de poner en claro todos estos procesos desde las teorías de la diferenciación social y de la representación política. Esta última se hace necesaria porque los citados procesos ocurren en un momento en el que estas sociedades están atravesando por una crisis radical de la estructura de relación -de comunicación- entre la sociedad civil y la esfera política democrática. Se traza así un esbozo de las complejas relaciones entre sociedad civil, cultura, religión y política en estas sociedades europeas occidentales.

Palabras clave: diferenciación social; representación política; homogeneización cultural; globalización cultural; re-creación cultural; diferenciación religión-política; diferenciación religión-cultura.

\section{Contemporary Transformations of the Relations between Politics, Culture and Religion in Western Europe. Some Notes on the Spanish Case}

\begin{abstract}
According to Tilly, two laws shaped the process of transformation undergone by Western European societies since the Peace of Westphalia until the end of the $20^{\text {th }}$ century: their increasing inner homogenisation and their growing heterogeneity between them. Cultural inner homogenisation affected, first, those ethnic groups living within the territories of the said states. The second phase of homogenisation impinged on those groups that immigrated after World War II. This process followed different models according to the country considered, but the 1973 oil crisis revealed their general lack of success. During the last quarter of the $20^{\text {th }}$ century and onwards, these European societies have been altered by two progressive and contradictory global logics: a process of cultural homogenisation at the world level (rather than society level) and a process of cultural re-creation led by those groups with an immigrant background, who have
\end{abstract}


reacted against their integration shortcomings by searching for new sources of social and personal esteem in their respective cultural and religious traditions. This paper seeks to clarify these processes from a social differentiation and political representation theory perspective. The latter becomes indispensable, as the said processes have happened in a context in which the structure of relations (i.e. communication) between civil society and the democratic political sphere have experienced a radical crisis. In this way, the complex relations that exist between civil society, culture, religion and politics in these Western European societies are depicted.

Key words: social differentiation; political representation; cultural homogenization; cultural globalization; cultural re-creation; religion-politics differentiation; religion-culture differentiation.

\title{
Referencia normalizada
}

Pérez-Agote, A. (2016): “Transformaciones contemporáneas de la relación entre política, cultura y religión en Europa occidental. Un apunte para el caso español”, Política y Sociedad, 53 (1), pp. 29-54.

Sumario: 1. El proceso histórico de homogeneización cultural y las cambiantes relaciones entre política, religión y cultura. 2. La crisis contemporánea de la homogeneidad cultural y religiosa. 3. Un apunte sobre el caso español. 4. Bibliografría.

\author{
$* * *$ \\ Agradecimientos \\ Para Pedro Ibarra, tras quinquenios de amistad.
}

\section{El proceso histórico de homogeneización cultural y las cambiantes relaciones entre política, religión y cultura}

Entre la Edad Media y nuestros días, las sociedades europeas occidentales han estado atravesadas por el proceso de construcción del Estado y por el proceso de nacionalización de éste. El primero de estos procesos es el de la construcción de una totalidad política dentro de un territorio definido por unas fronteras claras. Ello implica que esta unidad está claramente diferenciada de su entorno formado por otros Estados. Pero para que esto ocurra, en el interior de su territorio se dibuja otra diferenciación interna: la de un centro en relación con el resto de la realidad social del territorio, que constituye la periferia. De manera que el proceso de construcción del Estado está compuesto, en términos de la teoría de la diferenciación, de un doble momento: el de diferenciarse de los otros Estados y el de separarse un centro de una periferia. Según Charles Tilly, antes del siglo XVII, todos los Estados europeos gobernaban a sus miembros a través de intermediarios bastante autónomos. Esto significaba para los Estados una pérdida de recursos económicos. Para poder hacer frente a los gastos de la guerra, los Estados debieron suprimir los intermediarios, para dirigirse directamente a las comunidades y a los hogares habitados por los miembros. Y para hacer esto, cada Estado debió homogeneizar su población. Tilly nos recuerda la homogeneización de la población de los grandes Estados, como España, que expulsó a judíos, musulmanes y gitanos 